\title{
GENERAL STIELTJES MOMENT PROBLEMS FOR RAPIDLY DECREASING SMOOTH FUNCTIONS
}

\author{
RICARDO ESTRADA AND JASSON VINDAS
}

\begin{abstract}
We give (necessary and sufficient) conditions over a sequence $\left\{f_{n}\right\}_{n=0}^{\infty}$ of functions under which every generalized Stieltjes moment problem

$$
\int_{0}^{\infty} f_{n}(x) \phi(x) \mathrm{d} x=a_{n}, \quad n \in \mathbb{N},
$$

has solutions $\phi \in \mathcal{S}(\mathbb{R})$ with $\operatorname{supp} \phi \subseteq[0, \infty)$. Furthermore, we consider more general problems of this kind for measure or distribution sequences $\left\{f_{n}\right\}_{n=0}^{\infty}$. We also study vector moment problems with values in Fréchet spaces and multidimensional moment problems.
\end{abstract}

\section{INTRODUCTION}

The problem of moments, as its generalizations, is an important mathematical problem which has attracted much attention for more than a century. It was first raised and solved by Stieltjes for non-negative measures [24, 25]. Boas [1] and Pólya [21] showed later that given an arbitrary sequence $\left\{a_{n}\right\}_{n=0}^{\infty}$ there is always a function of bounded variation $F$ such that

$$
\int_{0}^{\infty} x^{n} \mathrm{~d} F(x)=a_{n}, \quad n \in \mathbb{N} .
$$

A major improvement to this result was achieved by Durán, who was able to show the existence of regular solutions to (1.1). He proved in [4] that every Stieltjes moment problem

$$
\int_{0}^{\infty} x^{n} \phi(x) \mathrm{d} x=a_{n}, \quad n \in \mathbb{N},
$$

admits a solution $\phi \in \mathcal{S}(0, \infty)$, that is, a solution in the Schwartz class of rapidly decreasing smooth functions $\mathcal{S}(\mathbb{R})$ with $\operatorname{supp} \phi \subseteq[0, \infty)$. The corresponding generalization for the strong moment problem has been given in [6]. Extensions of these results to vector-valued Stieltjes moment problems are also well-known [5, 7, 14]. Chung, Chung,

2010 Mathematics Subject Classification. Primary 30E05, 47A57, 44A60; Secondary 46F05.

Key words and phrases. Stieltjes moment problems; rapidly decreasing smooth solutions.

J. Vindas gratefully acknowledges support from Ghent University, through the BOF-grant number $01 \mathrm{~N} 01014$. 
and Kim, and more recently, Lastra and Sanz have considered moment problems with solutions in Gelfand-Shilov classes [3, 17, 18].

The problem that we are concerned with in this article is a general Stieltjes moment problem in which we replace the sequence of monomials $\left\{x^{n}\right\}_{n=0}^{\infty}$ in (1.2) by a rather general sequence of functions (or distributions) $\left\{f_{n}\right\}_{n=0}^{\infty}$. We are interested in conditions over $\left\{f_{n}\right\}_{n=0}^{\infty}$ that ensure the existence of solutions $\phi \in \mathcal{S}(0, \infty)$ to every infinite system of equations

$$
\int_{0}^{\infty} f_{n}(x) \phi(x) \mathrm{d} x=a_{n}, \quad n \in \mathbb{N}
$$

for a given arbitrary sequence $\left\{a_{n}\right\}_{n=0}^{\infty}$. In particular, we shall show that the following conditions on the asymptotic behavior of the primitives of the sequence suffice for the solvability of every (1.3):

Theorem 1.1. Let $\left\{f_{n}\right\}_{n=0}^{\infty}$ be a sequence of locally integrable functions on $[0, \infty)$ having at most polynomial growth at infinity. Then, every generalized moment problem (1.3) has solutions $\phi$ in $\mathcal{S}(0, \infty)$ if the sequence $\left\{f_{n}\right\}_{n=0}^{\infty}$ fulfills:

(i) $\int_{0}^{x} f_{n}(t) \mathrm{d} t=o\left(\int_{0}^{x} f_{n+1}(t) \mathrm{d} t\right)$ as $x \rightarrow \infty, n \in \mathbb{N}$.

(ii) For every $\alpha>0$ there are $N=N_{\alpha}$ and $\sigma=\sigma_{\alpha}>1$ such that

$$
\inf _{a \in[1, \sigma]}\left|\int_{0}^{a x} f_{N}(t) \mathrm{d} t\right|=\Omega\left(x^{\alpha}\right) \quad \text { as } x \rightarrow \infty .
$$

Theorem 1.1 is a general version of Durán's theorem quoted above. It also covers the case $f_{n}(x)=x^{\alpha_{n}}$ or generalized moment problems such as

$$
\int_{0}^{\infty} x^{\alpha_{n}} \sin \left(\frac{1}{x^{\beta}}\right) \phi(x) \mathrm{d} x=a_{n}, \quad n \in \mathbb{N} \quad(\beta>0),
$$

where $-1<\Re e \alpha_{0}<\Re e \alpha_{1}<\cdots<\Re e \alpha_{n} \rightarrow \infty$. The $\Omega$ in (1.4) stands for the HardyLittlewood symbol, namely, the negation of Landau's little $o$ symbol: $f(x)=\Omega(g(x))$ (as $x \rightarrow \infty$ ) means that there is a constant $C>0$ such that the inequality $|f(x)| \geq$ $C|g(x)|$ holds infinitely often for arbitrarily large values of $x$.

The assumptions on the sequence $\left\{f_{n}\right\}_{n=0}^{\infty}$ from Theorem 1.1 can be greatly relaxed. As we show, there is a corresponding result that applies for sequences of functions that might not be even locally Lebesgue integrable. In fact, we study in Section 3 the general moment problem for distribution sequences $\left\{f_{n}\right\}_{n=0}^{\infty}$. We provide in Theorem 3.1 a complete characterization of those distribution sequences for which all moment problems $a_{n}=\left\langle f_{n}, \phi\right\rangle, n \in \mathbb{N}$, have solutions $\phi \in \mathcal{S}(0, \infty)$. The notion of Cesàro admissibility, introduced in Section 3, plays a key role in our criterion for the existence of solutions in $\mathcal{S}(0, \infty)$ to generalized moment problems. In Section 4 , we specialize our results to the case of function sequences. 
We consider in Section 5 measure weighted moment problems of the form

$$
\int_{0}^{\infty} x^{\alpha_{n}} \phi(x) \mathrm{d} F(x)=a_{n}, \quad n \in \mathbb{N},
$$

for a (fixed) non-negative measure $\mathrm{d} F$ and a sequence of real exponents $\left\{\alpha_{n}\right\}_{n=0}^{\infty}$. Theorem 5.2 below gives necessary and sufficient conditions for the solvability of every (1.6) in $\mathcal{S}(0, \infty)$. Interestingly, our general considerations apply to show the existence of solutions to moment problems that could arise in quite different terms. For example, if $\left\{\alpha_{n}\right\}_{n=0}^{\infty}$ is an increasing sequence of real numbers tending to $\infty$, then, as follows from our results, every discrete moment problem

$$
\sum_{p \text { prime }} p^{\alpha_{n}} \phi(p)=a_{n}, \quad n \in \mathbb{N},
$$

admits solutions $\phi \in \mathcal{S}(0, \infty)$.

Section 7 is devoted to vector moment problems with values in a Fréchet space. Our analysis of the vector moment problem is based upon some results on the density of the set of solutions to moment problems, which will be obtained in Section 6. We conclude the article by studying moment problems in several variables in Section 8.

\section{Preliminaries}

We use the standard notation from distribution theory $[2,11,28]$. The space $\mathcal{S}_{+}^{\prime}$ denotes [28] the space of all tempered distributions with supports in the interval $[0, \infty)$. It can be canonically identified with the dual space of $\mathcal{S}_{+}$, where

$$
\mathcal{S}_{+}=\left\{\psi \in C^{\infty}[0, \infty): \psi=\varphi_{\left.\right|_{[0, \infty)}}, \text { for some } \varphi \in \mathcal{S}(\mathbb{R})\right\}
$$

Notice that $\mathcal{S}(0, \infty)$ is a closed subspace of $\mathcal{S}_{+}$, where, as in the Introduction, $\mathcal{S}(0, \infty)$ consists of those $\psi \in \mathcal{S}_{+}$such that $\psi^{(m)}(0)=0$ for every $m \in \mathbb{N}$.

We denote as $\mathcal{N}_{0}$ the annihilator of $\mathcal{S}(0, \infty)$ in $\mathcal{S}_{+}^{\prime}$. It is then clear that $\mathcal{N}_{0}$ consists of delta sums at the origin, that is, finite linear combinations of the Dirac delta $\delta$ and its derivatives.

We shall employ the notion of Cesàro behavior of distributions, introduced in [8] (see also $[11,13,20])$. We start with primitives of distributions [28]. Given $f \in \mathcal{S}_{+}^{\prime}$ and $m \in \mathbb{N}$, we denote as $f^{(-m)}$ the $m$-primitive of $f$ that satisfies $f^{(-m)} \in \mathcal{S}_{+}^{\prime}$. It can be expressed as the convolution [28]

$$
f^{(-m)}=f * \frac{x_{+}^{m-1}}{(m-1) !} .
$$

The Cesàro order growth symbols for distributions are defined as follows. Let $\alpha \in$ $\mathbb{R} \backslash\{-1,-2, \ldots\}$ and $m \in \mathbb{N}$, for $f \in \mathcal{S}_{+}^{\prime}$, we write

$$
f(x)=O\left(x^{\alpha}\right) \quad(\mathrm{C}, m), \quad x \rightarrow \infty,
$$


if $f^{(-m)}$ is a regular distribution (locally Lebesgue integrable) for large arguments and there is a polynomial $P$ of degree at most $m-1$ such that

$$
f^{(-m)}(x)=P(x)+O\left(x^{\alpha+m}\right) \quad \text { as } x \rightarrow \infty .
$$

Observe that if $\alpha>-1$ the polynomial in (2.1) is then irrelevant. The little $o$ symbol is defined in a similar fashion. When $f$ is locally Lebesgue integrable, the relation (2.1) reads as

$$
\frac{1}{x} \int_{0}^{x} f(t)\left(1-\frac{t}{x}\right)^{m-1} \mathrm{~d} t=\frac{Q(x)}{x^{m}}+O\left(x^{\alpha}\right),
$$

for some polynomial $Q$ of degree at most $m-1$. We also introduce the Hardy-Littlewood symbol $\Omega\left(x^{\alpha}\right)$ in the Cesàro sense. Thus, we define

$$
f(x)=\Omega\left(x^{\alpha}\right) \quad(\mathrm{C}, m), \quad x \rightarrow \infty,
$$

as the negation of $f(x)=o\left(x^{\alpha}\right) \quad(\mathrm{C}, m), x \rightarrow \infty$; in particular, if $\alpha>-1$ and $f^{(-m)}(x)$ is a function for large $x$, it just means that $f^{(-m)}(x)=\Omega\left(x^{\alpha+m}\right)$. Analogous definitions apply as $x \rightarrow 0^{+}$.

If we do not have to emphasize the role of $m$ in a Cesàro order relation we simply write $(\mathrm{C})$, which stands for $(\mathrm{C}, m)$ for some $m$. The growth order symbols can be used to define asymptotic relations and distributional evaluations in the Cesàro sense, see [11] for details.

\section{General moment problems for distribution SEQuences}

We study in this section the following general moment problem. Let $\left\{f_{n}\right\}_{n=0}^{\infty} \subset \mathcal{S}_{+}^{\prime}$ be a sequence of distributions. We seek conditions over $\left\{f_{n}\right\}_{n=0}^{\infty}$ such that every generalized moment problem, for a given arbitrary sequence $\left\{a_{n}\right\}_{n=0}^{\infty}$,

$$
\left\langle f_{n}, \phi\right\rangle=a_{n}, \quad n \in \mathbb{N},
$$

admits a solution $\phi \in \mathcal{S}(0, \infty)$.

We start with a natural condition over $\left\{f_{n}\right\}_{n=0}^{\infty}$. First, notice that if (3.1) is solvable for arbitrary sequences we must necessarily have

(P1') the distibutions $f_{0}, f_{1}, f_{2}, \ldots, f_{n}, \ldots$ are linearly independent.

Since we are interested in solutions to $(3.1)$ in $\mathcal{S}(0, \infty)$, one should assume that none of the $f_{n}$ is a delta sum at the origin; therefore, we assume the following relation between the sequence and the annihilator of $\mathcal{S}(0, \infty)$ :

(P1) No element of $\mathcal{N}_{0}$ is a linear combination of $f_{0}, f_{1}, f_{2}, \ldots, f_{n}, \ldots$.

Naturally (P1) implies (P1') since one considers the zero distribution is a delta sum with zero coefficients. 
A key property in our criterion for the existence of solutions to (3.1) is the notion of Cesàro admissibility, defined as follows. We shall say that the sequence $\left\{f_{n}\right\}_{n=0}^{\infty}$ is Cesàro admissible or that it satisfies the property (P2) if: There is an increasing sequence of integers $\left\{m_{j}\right\}_{j=0}^{\infty}$ such that for every $j \in \mathbb{N}$ and every $\alpha>0(\alpha \notin \mathbb{Z})$ there exists $\nu=\nu_{j, \alpha} \in \mathbb{N}$ such that if $N \geq \nu$

(P2)

$$
\left.\begin{array}{l}
\sum_{n=0}^{N} b_{n} f_{n}(x)=O\left(x^{\alpha}\right) \quad\left(\mathrm{C}, m_{j}\right), \quad x \rightarrow \infty, \\
\left(\sum_{n=0}^{N} b_{n} f_{n}^{\left(-m_{j}\right)}\right)_{\mid(0, \infty)} \in C(0, \infty), \text { and } \\
\sum_{n=0}^{N} b_{n} f_{n}(x)=O\left(x^{-\alpha}\right) \quad\left(\mathrm{C}, m_{j}\right), \quad x \rightarrow 0^{+}
\end{array}\right\} \Longrightarrow b_{\nu}=b_{\nu+1}=\cdots=b_{N}=0 .
$$

Note that (P2) is equivalent to: For each $m \in \mathbb{N}$ and $\alpha \in \mathbb{R}_{+} \backslash \mathbb{N}$ there exits $\nu=\nu_{\alpha, m} \in$ $\mathbb{N}$ such that if $N \geq \nu$ and $b_{N} \neq 0$, then

$$
\begin{gathered}
\sum_{n=0}^{N} b_{n} f_{n}(x)=\Omega\left(x^{\alpha}\right) \quad(\mathrm{C}, m), \quad x \rightarrow \infty, \quad \text { or } \\
\left(\sum_{n=0}^{N} b_{n} f_{n}^{(-m)}\right)_{\mid(0, \infty)} \notin C(0, \infty), \quad \text { or } \\
\sum_{n=0}^{N} b_{n} f_{n}(x)=\Omega\left(x^{-\alpha}\right) \quad(\mathrm{C}, m), \quad x \rightarrow 0^{+},
\end{gathered}
$$

holds. For example, the sequence of monomials $f_{n}(x)=x^{n}$ is Cesàro admissible, but so are $f_{n}(x)=\operatorname{Pf}\left(x^{-n}\right)$ and $f_{n}(x)=\delta^{(n)}(x-1), n \in \mathbb{N}$.

The ensuing theorem is the main result of this section.

Theorem 3.1. Every generalized moment problem (3.1) has a solution $\phi \in \mathcal{S}(0, \infty)$ if and only if the distribution sequence $\left\{f_{n}\right\}_{n=0}^{\infty}$ satisfies the properties $(\mathbf{P 1})$ and $(\mathbf{P 2})$.

In the proof of Theorem 3.1, we shall employ a result of Silva.

Lemma 3.2 (Silva [23]). Let $E$ be a Silva space, namely, $E$ is the inductive limit of an increasing sequence of Banach spaces $\left\{E_{n}\right\}_{n=0}^{\infty}$ where each inclusion mapping $E_{n} \rightarrow E_{n+1}$ is compact. Then, a linear subspace $X \subset E$ is closed if and only if $X \cap E_{n}$ is closed in each $E_{n}$.

We point out that the class of Silva spaces is precisely that of (DFS)-spaces (strong duals of Fréchet-Schwartz spaces). 
Proof of Theorem 3.1. Let $\mathbb{C}[[\xi]]$ be the space of formal power series in one indeterminate with the topology of convergence in each coefficient. Its dual is the space of polynomials in one-variable, denoted here as $\mathcal{P}$. That every generalized moment problem (3.1) has a solution $\phi \in \mathcal{S}(0, \infty)$ is equivalent to the surjectivity of the continuous linear mapping

$$
\Lambda: \mathcal{S}(0, \infty) \rightarrow \mathbb{C}[[\xi]]
$$

given by

$$
\Lambda(\psi)=\sum_{n=0}^{\infty} \mu_{n}(\psi) \xi^{n}, \quad \text { where } \mu_{n}(\psi)=\left\langle f_{n}, \psi\right\rangle .
$$

By the well-known criterion for surjectivity of continuous linear mappings between Fréchet spaces [26, Thm. 37.2, p. 382], the mapping $\Lambda$ is surjective if and only if its transpose

$$
\Lambda^{\top}: \mathcal{P} \rightarrow \mathcal{S}^{\prime}(0, \infty)=\mathcal{S}_{+}^{\prime} / \mathcal{N}_{0}
$$

is injective and has weakly* closed range. Since $\mathcal{S}_{+}^{\prime} / \mathcal{N}_{0}$ is Montel, it is reflexive, and there is therefore no distinction between weakly* closedness or strong closedness for its linear subspaces. The transpose of $\Lambda$ is easily seen to be given by

$$
\Lambda^{\top}\left(\sum_{n=0}^{N} b_{n} \xi^{n}\right)=\sum_{n=0}^{N} b_{n} f_{n \mid \mathcal{S}(0, \infty)} .
$$

It is then obvious that $\Lambda^{\top}$ is injective if and only if $(\mathbf{P} \mathbf{1})$ holds. Write $\pi$ for the quotient mapping $\pi: \mathcal{S}_{+}^{\prime} \rightarrow \mathcal{S}_{+}^{\prime} / \mathcal{N}_{0}$.

We first show the sufficience of $(\mathbf{P 1})$ and $(\mathbf{P} 2)$ for $\Lambda^{\top}$ to have closed range. Let $\mathcal{M}$ be the linear span of the $f_{0}, f_{1}, \ldots, f_{n}, \ldots$ in $\mathcal{S}_{+}^{\prime}$. Furthermore, the range of $\Lambda^{\top}$ is $\pi(\mathcal{M})$. We now use the fact that $\mathcal{S}_{+}^{\prime}$ is a Silva space. For its defining inductive sequence, we use the choice as in [28]. For each $n \in \mathbb{N}$, let $\mathcal{S}_{+, n}$ be the completion of $\mathcal{S}_{+}$in the norm

$$
\|\psi\|_{n}=\sup _{x \in[0, \infty), j \leq n}\left(1+x^{2}\right)^{n / 2}\left|\psi^{(j)}(x)\right| .
$$

Clearly, $\mathcal{S}_{+, n}$ consists of those functions $\psi \in C^{n}[0, \infty)$ such that $\lim _{x \rightarrow \infty} x^{n} \psi^{(j)}(x)=0$ for $0 \leq j \leq n$. Then, the injection $\mathcal{S}_{+, n}^{\prime} \rightarrow \mathcal{S}_{+, n+1}^{\prime}$ is compact and $\mathcal{S}_{+}^{\prime}=\bigcup_{n=0}^{\infty} \mathcal{S}_{+, n}^{\prime}$, topologically. Moreover, denoting $\mathcal{S}_{0, n}^{\prime}=\pi\left(\mathcal{S}_{+, n}^{\prime}\right)$, we have that $\mathcal{S}^{\prime}(0, \infty)=\bigcup_{n=0}^{\infty} \mathcal{S}_{0, n}^{\prime}$ topologically and each $\mathcal{S}_{0, n}^{\prime} \rightarrow \mathcal{S}_{0, n+1}^{\prime}$ is also compact. By Lemma 3.2, $\pi(\mathcal{M})$ is closed if and only if $\pi(\mathcal{M}) \cap \mathcal{S}_{0, n}^{\prime}$ is closed in $\mathcal{S}_{0, n}^{\prime}$ for each $n$. Since finite dimensional subspaces are always closed, the latter will be a consequence of the following claim:

Claim 1. Cesàro admissibility implies that $\pi(\mathcal{M}) \cap \mathcal{S}_{0, n}^{\prime}$ is finite dimensional for each $n \in \mathbb{N}$. 
Indeed, for each $k \in \mathbb{N}$, set

$$
\mathcal{X}_{k}=\left\{\sum_{n=0}^{k} b_{n} f_{n}: b_{n} \in \mathbb{C}\right\} \oplus \mathcal{N}_{0} \subset \mathcal{M} \oplus \mathcal{N}_{0} .
$$

Let $\left\{m_{j}\right\}_{j=0}^{\infty}$ be the sequence from (P2). We show that for $p=m_{j}-2>0$, there is $k=k_{j}$ such that $\left(\mathcal{M} \oplus \mathcal{N}_{0}\right) \cap \mathcal{S}_{+, p}^{\prime} \subseteq \mathcal{X}_{k}$, whence the claim would follow. Suppose that $g=\sum_{n=0}^{N}\left(b_{n} f_{n}+c_{n} \delta^{(n)}\right) \in \mathcal{S}_{+, p}^{\prime}$. Find $\nu$ such that $(\mathbf{P 2})$ holds for $j$ and $\alpha=2 p+3 / 2$. Observe that $\varphi_{x}(u)=(x-u)_{+}^{p+1} \in \mathcal{S}_{+, p}$. Thus,

$$
g^{\left(-m_{j}\right)}(x)=g^{(-p-2)}(x)=g * \frac{x_{+}^{p+1}}{(p+1) !}=\frac{1}{(p+1) !}\left\langle g(u), \varphi_{x}(u)\right\rangle
$$

is a continuous function (on the whole $[0, \infty)$ ) and

$$
\begin{aligned}
\left|g^{(-p-2)}(x)\right| & \leq \frac{\|g\|_{\mathcal{S}_{+, p}^{\prime}}\left\|\varphi_{x}\right\|_{p}}{(p+1) !}=O\left(x^{p+1} \sup _{u \in[0, x), j \leq p}\left(|u|^{p}+1\right)(1-u / x)^{p+1-j}\right) \\
& =O\left(x^{2 p+1}\right) .
\end{aligned}
$$

Hence, $\sum_{n=0}^{N} b_{n} f_{n}(x)=O\left(x^{\alpha}\right)\left(\mathrm{C}, m_{j}\right), x \rightarrow \infty, \sum_{n=0}^{N} b_{n} f_{n}^{\left(-m_{j}\right)}(x)$ is continuous for $x \in(0, \infty)$, and $\sum_{n=0}^{N} b_{n} f_{n}(x)=O\left(x^{-\alpha}\right)\left(\mathrm{C}, m_{j}\right), x \rightarrow 0^{+}$. We obtain from (P2) that $b_{n}=0$ for $n \geq \nu$. Thus, $g \in \mathcal{X}_{\nu-1}$. The claim has been established.

Conversely, assume that $\Lambda^{\top}$ is injective and has weakly* closed range. As already pointed out, the range $\Lambda^{\top}(\mathcal{P})$ is thus strongly closed because $\mathcal{S}_{+}^{\prime} / \mathcal{N}_{0}$ is reflexive (in fact a (DFS)-space). We have already noticed that (P1) must necessarily hold. To show (P2), we first establish that $\Lambda^{\top}$ is an isomorphism into its image. Pták's theory $[16,22]$ applies to show that $\Lambda^{\top} \mathcal{P} \rightarrow \Lambda^{\top}(\mathcal{P})$ is open if we verify that $\mathcal{P}$ is fully complete ( $B$-complete in the sense of Pták) and that $\Lambda^{\top}(\mathcal{P})$ is barreled. It is well known [22, p. 123] that the strong dual of a reflexive Fréchet space is fully complete, so $\mathcal{P}$, as a (DFS)-space, is fully complete. Now, a closed subspace of a (DFS)-space must itself be a (DFS)-space. Since $\mathcal{S}_{+}^{\prime} / \mathcal{N}_{0}$ is a (DFS)-space, we obtain that $\Lambda^{\top}(\mathcal{P})$ is also a (DFS)-space and hence barreled.

Suppose now that (P2) were false. Then, there are $j$ and $\alpha>0$ such that $g_{n}(x)=$ $\sum_{\nu=0}^{n} b_{\nu, n} f_{\nu}(x)=O\left(x^{\alpha}\right)(\mathrm{C}, j), x \rightarrow \infty, g_{n}(x)=O\left(x^{-\alpha}\right)(\mathrm{C}, j), x \rightarrow 0^{+}$, and $g_{n}^{(-j)}(x) \in$ $C(0, \infty)$, with $b_{n, n} \neq 0$ for infinitely many indices $n$. If $p \geq \max \{\alpha+j+2\}$, we conclude that there is an increasing sequence of indices $n_{0}<n_{1}<\cdots<n_{k}<\ldots$ such that $\left\{\pi\left(g_{n_{k}}\right)\right\}_{k=0}^{\infty} \subset \mathcal{S}_{0, p}^{\prime}$. Let $h_{n_{k}}=\sum_{\nu=0}^{n_{k}}\left(b_{\nu, n_{k}} /\left\|\pi\left(g_{n_{k}}\right)\right\|_{\mathcal{S}_{0, p}^{\prime}}\right) \pi\left(f_{\nu}\right)=\sum_{\nu=0}^{n_{k}} a_{\nu, n_{k}} \pi\left(f_{\nu}\right)$ with $a_{n_{k}, n_{k}} \neq 0$. Then $\left\{h_{n_{k}}\right\}_{k=0}^{\infty}$ is bounded in $\mathcal{S}_{0}^{\prime}$ because of the continuity of the inclusion mapping $\mathcal{S}_{0, p}^{\prime} \rightarrow \mathcal{S}^{\prime}(0, \infty)$. We deduce then that $\left\{h_{n_{k}}\right\}_{k=0}^{\infty}$ is a bounded set of $\Lambda^{T}(\mathcal{P})$ and since $\Lambda^{\top}$ is open, we should have that the set of polynomials $\left\{\sum_{\nu=0}^{n_{k}} a_{\nu, n_{k}} \xi^{\nu}\right\}_{k=0}^{\infty}$ is 
bounded in $\mathcal{P}$ as well. But the latter can only hold if there is $k_{0}$ such that $a_{\nu, n_{k}}=0$ for all indices $n_{k} \geq n_{k_{0}}$ and $\nu \geq n_{k_{0}}$, which produces a contradiction. This completes the proof of the theorem.

Let us discuss a simple example to illustrate Theorem 3.1.

Example 3.3 (The generalized Borel problem). Let $\left\{k_{n}\right\}_{n=0}^{\infty}$ be sequence of natural numbers with $k_{n} \rightarrow \infty$ and let $\left\{x_{n}\right\}_{n=0}^{\infty}$ be a sequence of positive real numbers such that all pairs $\left(k_{n}, x_{n}\right)$ are distinct. The distribution sequence $\left\{f_{n}\right\}_{n=0}^{\infty}$ given by $f_{n}(x)=$ $\delta^{\left(k_{n}\right)}\left(x-x_{n}\right)$ satisfies $(\mathbf{P 1})$ and $(\mathbf{P} 2)$. Consequently, Theorem 3.1 gives that

$$
\phi^{\left(k_{n}\right)}\left(x_{n}\right)=a_{n}, \quad n \in \mathbb{N},
$$

is always solvable in $\mathcal{S}(0, \infty)$. If $\left\{x_{n}\right\}_{n=0}^{\infty}$ stays on a fixed compact subset of $(0, \infty)$, multiplying by a cut-off function, we can in fact find solutions to (3.2) that belong to $\mathcal{D}(0, \infty)$. In particular, choosing $x_{n}=x_{0}$ to be constant and $k_{n}=n$ the sequence of all natural numbers, we recover the well known fact that

$$
\phi^{(n)}\left(x_{0}\right)=a_{n}, \quad n \in \mathbb{N} .
$$

has solution $\phi \in \mathcal{D}(\mathbb{R})$. Performing a translation, one easily sees that one may take an arbitrary $x_{0} \in \mathbb{R}$ in (3.3), that is, every classical Borel problem has a solution $\phi \in \mathcal{D}(\mathbb{R})$.

Theorem 3.1 can be generalized to distribution two-sided sequences $\left\{f_{n}\right\}_{n \in \mathbb{Z}}$. In fact, consider

$$
\left\langle f_{n}, \phi\right\rangle=a_{n}, \quad n \in \mathbb{Z}
$$

If we set for $n \in \mathbb{N}$

$$
c_{n}=\left\{\begin{array}{ll}
a_{n / 2} & \text { if } n \text { is even } \\
a_{-(n+1) / 2} & \text { if } n \text { is odd }
\end{array} \text { and } \quad g_{n}= \begin{cases}f_{n / 2} & \text { if } n \text { is even } \\
f_{-(n+1) / 2} & \text { if } n \text { is odd },\end{cases}\right.
$$

then the moment problem (3.4) is equivalent to $\left\langle g_{n}, \phi\right\rangle=c_{n}, n \in \mathbb{N}$, while that (P1) and (P2) hold for $\left\{g_{n}\right\}_{n=0}^{\infty}$ translates into:

$\left(\mathbf{P} \mathbf{1}^{*}\right)$ no element of $\mathcal{N}_{0}$ is a linear combination of $\ldots, f_{-2}, f_{-1}, f_{0}, f_{1}, f_{2}, \ldots$, and $\left(\mathbf{P} 2^{*}\right)$ there is an increasing sequence of integers $\left\{m_{j}\right\}_{j=0}^{\infty}$ such that for each $j \in \mathbb{N}$ and each $\alpha>0$ there exists $\nu=\nu_{j, \alpha} \in \mathbb{N}$ such that if $N \geq \nu: \sum_{n=-N}^{N} b_{n} f_{n}(x)=$ $O\left(x^{\alpha}\right) \quad\left(\mathrm{C}, m_{j}\right), x \rightarrow \infty, \sum_{n=-N}^{N} b_{n} f_{n}^{\left(-m_{j}\right)}(x)$ is continuous for $x \in(0, \infty)$ and $\sum_{n=-N}^{N} b_{n} f_{n}(x)=O\left(x^{-\alpha}\right) \quad\left(\mathrm{C}, m_{j}\right), x \rightarrow 0^{+}$, imply $b_{n}=0$ for every $|n| \geq \nu$.

We thus obtain,

Corollary 3.4. Let $\left\{f_{n}\right\}_{n \in \mathbb{Z}} \subset \mathcal{S}_{+}^{\prime}$. Every (3.4) admits a solution $\phi \in \mathcal{S}(0, \infty)$ if and only if the conditions $\left(\mathbf{P} \mathbf{1}^{*}\right)$ and $\left(\mathbf{P 2}{ }^{*}\right)$ are satisfied. 
We end this section with an example.

Example 3.5. The so called strong moment problem,

$$
a_{n}=\int_{0}^{\infty} x^{n} \phi(x) \mathrm{d} x, \quad n \in \mathbb{Z},
$$

was studied and solved in [6] for $\phi \in \mathcal{S}(0, \infty)$. Using Corollary 3.4, we can strengthen the main result of [6] as follows. Let $\left\{\alpha_{n}\right\}_{n \in \mathbb{Z}}$ be a two-sided sequence. Then, every strong moment problem

$$
a_{n}=\int_{0}^{\infty} x^{\alpha_{n}} \phi(x) \mathrm{d} x, \quad n \in \mathbb{Z},
$$

is solvable in $\mathcal{S}(0, \infty)$ if and only if all elements of the sequence are distinct and $\left|\Re e \alpha_{n}\right| \rightarrow \infty$ as $|n| \rightarrow \infty$.

Remark 3.6. The simple reduction explained above for moment problems with twosided sequences also applies to all results from the next sections, we will therefore omit any comment concerning two-sided sequences in the sequel.

\section{Moment PRoblems With FUnCtion SEQUEnCES}

We now focus our attention on function sequences. Throughout this section $\left\{f_{n}\right\}_{n=0}^{\infty} \subset$ $\mathcal{S}_{+}^{\prime}$ stands for a sequence such that each $f_{n}$ is a non-identically zero locally integrable ${ }^{1}$ function with continuous primitives ${ }^{2}$ on $(0, \infty)$. Note that we allow $f_{n}$ not being integrable near $x=0$. The distributional evaluation $\left\langle f_{n}, \varphi\right\rangle$ for $\phi \in \mathcal{S}(0, \infty)$ can be always written [27] as a Cesàro integral and thus we may rewrite in this case the moment problem (3.1) as

$$
\int_{0}^{\infty} \phi(x) f_{n}(x) \mathrm{d} x=a_{n} \quad(\mathrm{C}), \quad n \in \mathbb{N},
$$

Naturally, if each $f_{n}$ has at most polynomial growth, then the Cesàro integrals in (4.1) can be replaced by ordinary integrals.

Theorem 1.1 gives already a complete characterization of those $\left\{f_{n}\right\}_{n=0}^{\infty}$ for which every moment problem is solvable in $\mathcal{S}(0, \infty)$. Note that $\left(\mathbf{P} \mathbf{1}^{\prime}\right)$ for $\left\{f_{n}\right\}_{n=0}^{\infty}$ becomes equivalent to $(\mathbf{P 1})$. On the other hand, (P2) forces the linear combinations of the

\footnotetext{
${ }^{1}$ All results from this section are valid for locally distributionally integrable functions in the sense of the authors [12], and in particular for sequences of locally Denjoy-Perron-Henstock or Lebesgue integrable functions on $(0, \infty)$.

${ }^{2}$ If $f_{n}$ is locally Denjoy-Perron-Henstock or Lebesgue integrable, its primitive is of course continuous. The primitives of distributional integrable functions are Łojasiewicz functions but in general they may be discontinuous [12], whence this assumption.
} 
primitives of the sequence to have the ensuing growth property: For each $m \in \mathbb{N}$ and $\alpha \in \mathbb{R}_{+} \backslash \mathbb{N}$ there exits $\nu=\nu_{\alpha, m} \in \mathbb{N}$ such that if $N \geq \nu$ and $b_{N} \neq 0$, then

$$
\sum_{n=0}^{N} b_{n} f_{n}^{(-m)}(x)=\Omega\left(x^{\alpha}\right) \quad \text { as } x \rightarrow \infty \quad \text { or } \quad \sum_{n=0}^{N} b_{n} f_{n}^{(-m)}(x)=\Omega\left(x^{-\alpha}\right) \quad \text { as } x \rightarrow 0^{+} .
$$

Since all primitives of $f_{n}$ are continuous on $(0, \infty)$, the latter property is actually equivalent to condition (P2).

The next theorem tells that if for a fixed $m$ one slightly strengthens these two conditions, then one obtains, together with linear independence, a useful criterion for the solvability of arbitrary moment problems (4.1).

Theorem 4.1. Let $m \geq 1$. Suppose that $\left\{f_{n}\right\}_{n=0}^{\infty}$ satisfies (P1') and has the following property:

(P3) For any given $\alpha>0$ there is $\nu=\nu_{\alpha} \in \mathbb{N}$ such that if $N \geq \nu$ and $b_{N} \neq 0$, then one can find $\sigma=\sigma_{N}>1$ such that

$$
\begin{aligned}
& \inf _{a \in[1, \sigma]}\left|\sum_{n=0}^{N} b_{n} f_{n}^{(-m)}(a x)\right|=\Omega\left(x^{\alpha}\right) \quad \text { as } x \rightarrow \infty, \\
& \text { or } \rightarrow \inf _{a \in[1, \sigma]}\left|\sum_{n=0}^{N} b_{n} f_{n}^{(-m)}(a x)\right|=\Omega\left(x^{-\alpha}\right) \quad \text { as } x \rightarrow 0^{+} .
\end{aligned}
$$

Then every generalized moment problem (4.1) is solvable in $\mathcal{S}(0, \infty)$. If additionally each $f_{n}$ is a Darboux function ${ }^{3}$ (i.e., has the intermediate value property), then (4.3) might be replaced by

$$
\begin{aligned}
& \inf _{a \in[1, \sigma]}\left|\sum_{n=0}^{N} b_{n} f_{n}(a x)\right|=\Omega\left(x^{\alpha}\right) \quad \text { as } x \rightarrow \infty, \\
& \quad \text { or } \\
& \inf _{a \in[1, \sigma]}\left|\sum_{n=0}^{N} b_{n} f_{n}(a x)\right|=\Omega\left(x^{-\alpha}\right) \quad \text { as } x \rightarrow 0^{+} .
\end{aligned}
$$

Proof. In view of Theorem 1.1, we only need to show that (P3) ensures the validity of (P2). Suppose that (P3) is satisfied but (P2) does not hold. Then, one can find $\alpha>j \geq 1(\alpha \notin \mathbb{N})$ and a sequence $g_{k}=\sum_{n=0}^{N_{k}} b_{n, N_{k}} f_{n}$ such that $b_{N_{k}, N_{k}} \neq 0$ and either

\footnotetext{
${ }^{3}$ Every continuous function is of course a Darboux function. More generally, if each $f_{n}$ is a Eojasiewicz function it must have the Darboux property [12, 19].
} 
$g_{k}^{(-j-m)}(x)=o\left(x^{\alpha+j}\right)$ as $x \rightarrow \infty$ or $g_{k}^{(-j-m)}(x)=o\left(x^{j-\alpha}\right)$ as $x \rightarrow 0^{+}$. The latter implies that, for each $\phi \in \mathcal{S}(0, \infty)$,

$$
\lim _{\lambda \rightarrow \infty} \frac{1}{\lambda^{\alpha}} \int_{0}^{\infty} g_{k}^{(-m)}(\lambda x) \phi(x) \mathrm{d} x=0 \quad \text { or } \lim _{\lambda \rightarrow 0^{+}} \lambda^{\alpha} \int_{0}^{\infty} g_{k}^{(-m)}(\lambda x) \phi(x) \mathrm{d} x=0 .
$$

Let $\nu$ be the integer corresponding to $\alpha$ in (P3). Fix $k$ and $\sigma>1$. Taking a nonnegative test function $\phi$ with $\operatorname{supp} \phi \subset[1, \sigma]$ and $\int_{1}^{\sigma} \phi(x) \mathrm{d} x=1$ in (4.5) and applying the mean-value theorem [12, Sect. 11], we obtain that for each $\lambda$ one can find $a_{\lambda} \in(1, \sigma)$ such that either

$$
\lim _{\lambda \rightarrow \infty} \frac{g_{k}^{(-m)}\left(\lambda a_{\lambda}\right)}{\lambda^{\alpha}}=0 \quad \text { or } \quad \lim _{\lambda \rightarrow \infty} \frac{g_{k}^{(-m)}\left(a_{\lambda} / \lambda\right)}{\lambda^{\alpha}}=0 .
$$

On the other hand, (P3) tells us that if $N_{k}>\nu$ there are $\sigma>1$, a sequence $\lambda_{n} \rightarrow \infty$, and $C>0$ such that either $\left|g_{k}^{(-m)}\left(\lambda_{n} a\right)\right| \geq C \lambda_{n}^{\alpha}$ or $\left|g_{k}^{(-m)}\left(a / \lambda_{n}\right)\right| \geq C \lambda_{n}^{\alpha}$ for all $a \in[1, \sigma]$ and $n \in \mathbb{N}$, contradicting (4.6).

Note that integration by parts in (4.5) leads to

$$
\text { either } \lim _{\lambda \rightarrow \infty} \frac{1}{\lambda^{\alpha-m}} \int_{0}^{\infty} g_{k}(\lambda x) \phi(x) \mathrm{d} x=0 \quad \text { or } \lim _{\lambda \rightarrow 0^{+}} \lambda^{\alpha+m} \int_{0}^{\infty} g_{k}(\lambda x) \phi(x) \mathrm{d} x=0 \text {. }
$$

If we assume that each $f_{n}$ is a Darboux function, then so is each $g_{k}$. The mean-value theorem still applies ${ }^{4}$ to prove that assuming that (P2) fails is contradictory with (4.4). The result has been established.

It should be noticed that Theorem 1.1, stated in the Introduction, immediately follows by taking $m=1$ in Theorem 4.1 .

Next, we are interested in weigthed moment problems of the form

$$
\int_{0}^{\infty} x^{\alpha_{n}} f(x) \phi(x) \mathrm{d} x=a_{n} \quad(\mathrm{C}), \quad n \in \mathbb{N}
$$

where $f \in \mathcal{S}_{+}^{\prime}$ is a (non-identically zero) locally integrable function (with continuous primitive) on $(0, \infty)$. We point out that $(\mathbf{P} 1)$ holds if and only if $\left\{\alpha_{n}\right\}_{n=0}^{\infty}$ consists of distinct complex numbers. We will prove that (P2) forces the sequence $\left\{\alpha_{n}\right\}_{n=0}^{\infty}$ to stay off vertical strips except for finitely many terms.

Theorem 4.2. Let $\left\{\alpha_{n}\right\}_{n=0}^{\infty}$ be a sequence of distinct complex numbers with the property there is $n_{0}$ such that $\Re e \alpha_{n} \neq \Re e \alpha_{m}$ for all distinct $n, m>n_{0}$. Then, every moment problem (4.7) is solvable in $\mathcal{S}(0, \infty)$ if and only if

$$
\lim _{n \rightarrow \infty}\left|\Re e \alpha_{n}\right|=\infty
$$

and

\footnotetext{
${ }^{4}$ See the proof of [12, Prop. 11.1].
} 
(I) if $\lim _{n \rightarrow \infty} \Re e \alpha_{n}=\infty$, then for each $m \in \mathbb{N}$ the $m$-primitive of $f$ satisfies

$$
-\infty<\limsup _{x \rightarrow \infty} \frac{\log \left|f^{(-m)}(x)-P(x)\right|}{\log x},
$$

for each polynomial $P$ of degree at most $m-1$;

(II) if $\lim _{n \rightarrow \infty} \Re e \alpha_{n}=-\infty$, then for each $m \in \mathbb{N}$.

$$
\infty<\limsup _{x \rightarrow 0^{+}} \frac{\log \left|f^{(-m)}(x)-P(x)\right|}{|\log x|},
$$

for each polynomial $P$ of degree at most $m-1$;

(III) if $\liminf _{n \rightarrow \infty} \Re e \alpha_{n}=-\infty$ and $\limsup \Re e \alpha_{n}=\infty$, then for each $m \in \mathbb{N}$ both (4.9) and (4.10) hold for each polynomial $P$ of degree at most $m-1$.

Proof. For each $n$, find $f_{n} \in \mathcal{S}_{+}^{\prime}$ such that $f_{n}(x)=x^{\alpha_{n}} f(x)$ for $x \in(0, \infty)$. It is clearly that the sequence $\left\{f_{n}\right\}_{n=0}^{\infty}$ is linearly independent on $(0, \infty)$ and consequently $\mathcal{N}_{0}$ has trivial intersection with the linear span of $\left\{f_{n}\right\}_{n=0}^{\infty}$.

Assume that every moment problem (4.7) is solvable in $\mathcal{S}(0, \infty)$. Fix $M>0$ and set $A_{M}=\left\{n \in \mathbb{N}:-M \leq \Re e \alpha_{n} \leq M\right\}$. Since $f \in \mathcal{S}_{+}^{\prime}$, there is $m \geq 1$ and $\theta>m$ such that $f^{(-m)} \in C[0, \infty), f^{(-m)}(x)=O\left(x^{\theta}\right)$ as $x \rightarrow \infty$, and $f^{(-m)}(x)=O\left(x^{M+1 / 2}\right)$ as $x \rightarrow 0^{+}$. On the other hand (cf. [11, Eq. (6.53), p. 302] or [2, Lemma 1.3]),

$$
f_{n}^{(-m)}=\sum_{k=0}^{m}(-1)^{k} k !\left(\begin{array}{c}
m \\
k
\end{array}\right)\left(\begin{array}{c}
\alpha_{n} \\
k
\end{array}\right)\left(x^{\alpha_{n}-k} f^{(-m)}\right)^{(-k)}+P \quad \text { on }(0, \infty) .
$$

where, $P(x)$ is a polynomial of degree at most $m-1$. So, if $n \in A_{M}$, one has that $f_{n}^{(-m)}(x)=O\left(x^{\theta+M}\right)$ as $x \rightarrow \infty$ and $f_{n}^{(-m)}(x)=Q(x)+O\left(x^{1 / 2}\right)$ as $x \rightarrow 0^{+}$for some polynomial $Q$ of degree at most $m-1$. In particular $f_{n}^{(-m)} \in C(0, \infty), f_{n}(x)=O\left(x^{\alpha}\right)$ $(\mathrm{C}, m), x \rightarrow \infty$, and $f_{n}=O\left(x^{-\alpha}\right)(\mathrm{C}, m), x \rightarrow 0^{+}$, for $\alpha \geq \max \{\theta+M-m, m-1 / 2\}$. The property (P2) implies that this cannot hold for infinitely many $n$, so that $A_{M}$ must be finite.

Suppose that $\lim _{n \rightarrow \infty} \Re e \alpha_{n}=\infty$ but that (4.9) is false for some $m$, i.e., $f^{(-m)}(x)=$ $Q(x)+o\left(x^{-\alpha_{n}}\right)$ as $x \rightarrow \infty$, for every $n>0$, where $Q$ is a polynomial of degree less than $m$. Since

$$
0=\sum_{k=0}^{m}(-1)^{k} k !\left(\begin{array}{c}
m \\
k
\end{array}\right)\left(\begin{array}{c}
\alpha_{n} \\
k
\end{array}\right)\left(x^{\alpha_{n}-k} Q\right)^{(m-k)} \quad \text { on }(0, \infty),
$$

the formula (4.10) then yields $f_{n}^{(-m)} \in C(0, \infty)$ and $f_{n}(x)=O(1)(\mathrm{C}, m)$ as $x \rightarrow \infty$, for each $n$. Furthermore, since $\Re e \alpha_{n}$ is bounded from below, we also get $f_{n}(x)=O\left(x^{-\alpha}\right)$ 
$(\mathrm{C}, m$ ), for some $\alpha$ (and a possibly enlarged $m$ ), whence we deduce that (4.2) (and hence (P2)) cannot hold. The cases (II) and (III) can be treated in a similar fashion.

An analogous argument, with aid of the relation (cf. [2, Lemma 1.3])

$$
f^{(-m)}=\sum_{k=0}^{m}(-1)^{k} k !\left(\begin{array}{c}
m \\
k
\end{array}\right)\left(\frac{g_{N}^{(-m)}}{F_{N}^{(k)}}\right)^{(-k)}+Q,
$$

where $F_{N}(x)=\sum_{n=0}^{N} b_{n} x^{\alpha_{n}}, g_{N}(x)=\sum_{n=0}^{N} b_{n} f_{n}$, and $Q$ a certain polynomial (depending on $F_{N}$ ) of degree at most $m-1$, shows that the conditions are sufficient for (P2).

Employing the same method as in proof of Theorem 4.1, one deduces:

Proposition 4.3. Let $\left\{\alpha_{n}\right\}_{n=0}^{\infty}$ be a sequence of distinct complex numbers with the property there is $n_{0}$ such that $\Re e \alpha_{n} \neq \Re e \alpha_{m}$ for all distinct $n, m>n_{0}$. Suppose that (4.8) holds and there are $m \geq 1$ and $\sigma>1$ such that

(I) if $\lim _{n \rightarrow \infty} \Re e \alpha_{n}=\infty$, then

$$
-\infty<\limsup _{x \rightarrow \infty} \frac{\log \inf _{a \in[1, \sigma]}\left|f^{(-m)}(a x)-P(a x)\right|}{\log x}
$$

for each polynomial $P$ of degree at most $m-1$;

(II) if $\lim _{n \rightarrow \infty} \Re e \alpha_{n}=-\infty$, then

$$
\liminf _{x \rightarrow 0^{+}} \frac{\log \inf _{a \in[1, \sigma]}\left|f^{(-m)}(a x)-P(a x)\right|}{|\log x|}<\infty,
$$

for each polynomial $P$ of degree at most $m-1$;

(III) if $\liminf _{n \rightarrow \infty} \Re e \alpha_{n}=-\infty$ and $\limsup _{n \rightarrow \infty} \Re e \alpha_{n}=\infty$, then both (4.12) and (4.13)) hold for each polynomial $P$ of degree at most $m-1$.

Then every moment problem (4.7) has solutions $\phi \in \mathcal{S}(0, \infty)$. If $f$ is a Darboux function, one may take $m=0$ (so that $P=0$ ) in (4.12) and (4.13).

\section{The MEAsure Weighted MOMENT PROBLEM}

We consider here the measure weighted moment problem

$$
\int_{0}^{\infty} x^{\alpha_{n}} \phi(x) \mathrm{d} F(x)=a_{n}, \quad n \in \mathbb{N},
$$


where we assume that $\left\{\alpha_{n}\right\}_{n=0}^{\infty}$ is sequence of real numbers and $F$ is a function of local bounded variation on $(0, \infty)$ that satisfies

$$
\int_{0}^{1} x^{\sigma_{1}}|\mathrm{~d} F|(x)<\infty
$$

and

$$
\int_{1}^{\infty} x^{-\sigma_{1}}|\mathrm{~d} F|(x)<\infty
$$

for some $\sigma_{1}>0$, where $|\mathrm{d} F|$ is the total variation measure of $\mathrm{d} F$. This ensures $\mathrm{d} F \in \mathcal{S}^{\prime}(0, \infty)$.

We start with a necessary condition for the solvability of (5.1).

Proposition 5.1. Suppose that every moment problem (5.1) has solutions $\phi \in \mathcal{S}(0, \infty)$, then the numbers $\alpha_{n}$ are distinct, $\left|\alpha_{n}\right| \rightarrow \infty$ and there is $\sigma_{0}>0$ such that

(I) if $\lim _{n \rightarrow \infty} \alpha_{n}=\infty$, then

$$
\int_{1}^{\infty} x^{-\sigma_{0}}|\mathrm{~d} F|(x)=\infty
$$

(II) if $\lim _{n \rightarrow \infty} \alpha_{n}=-\infty$, then

$$
\int_{0}^{1} x^{\sigma_{0}}|\mathrm{~d} F|(x)=\infty
$$

(III) if $\liminf _{n \rightarrow \infty} \alpha_{n}=-\infty$ and $\limsup _{n \rightarrow \infty} \alpha_{n}=\infty$, then both (5.4) and (5.5) hold.

Proof. Let $f_{n}$ be an extension of $x^{\alpha_{n}} \mathrm{~d} F(x)$ to $\mathcal{S}_{+}^{\prime}$. Since $(\mathbf{P} 1)$ holds, the $\alpha_{n}$ 's should be distinct. If $\left|\alpha_{n}\right| \leq M$ for all $n \in \mathbb{N}$, using the assumptions (5.2) and (5.3), we would have

$$
\left|\sum_{n=0}^{N} b_{n} \int_{1}^{x} t^{\alpha_{n}} \mathrm{~d} F(t)\right|=O\left(x^{M+\sigma_{1}}\right) \quad \text { as } x \rightarrow \infty
$$

and

$$
\left|\sum_{n=0}^{N} b_{n} \int_{1}^{x} t^{\alpha_{n}} \mathrm{~d} F(t)\right|=O\left(x^{-M-\sigma_{1}}\right) \quad \text { as } x \rightarrow 0^{+},
$$

contradicting (P2) for $\left\{f_{n}\right\}_{n=0}^{\infty}$. If $\alpha_{n} \rightarrow \infty$, a bound (5.6) must hold because $\alpha_{n}$ is bounded from below. If (5.4) were not valid, then we would have $\int_{1}^{\infty} t^{\alpha_{n}}|\mathrm{~d} F|(t)<\infty$ for all $n \in \mathbb{N}$, leading to

$$
\left|\sum_{n=0}^{N} b_{n} \int_{1}^{x} t^{\alpha_{n}} \mathrm{~d} F(t)\right|=O(1), \quad x \geq 1 .
$$


This again contradicts (P2). The cases (II) and (III) can be treated similarly.

Note that either (5.4) or (5.5) always yields that the support of $\mathrm{d} F$ is an infinite subset of $(0, \infty)$. If additionally the $\alpha_{n}$ 's are distinct, the latter implies $(\mathbf{P} \mathbf{1})$ for any distribution sequence $\left\{f_{n}\right\}_{n=0}^{\infty}$ of $\mathcal{S}_{+}^{\prime}$ that extends $x^{\alpha_{n}} \mathrm{~d} F(x)$, that is, $f_{n}(x)=x^{\alpha_{n}} \mathrm{~d} F(x)$ on $(0, \infty)$.

We give the converse of Proposition 5.1 for non-negative measures. In fact, the next theorem gives a complete characterization of those non-negative measures $\mathrm{d} F$ for which (5.1) is always solvable in $\mathcal{S}(0, \infty)$.

Theorem 5.2. Let $F$ be non-decreasing on $(0, \infty)$ satisfying (5.2) and (5.3), and let $\left\{\alpha_{n}\right\}_{n=0}^{\infty}$ be a sequence of distinct real numbers. Then, every moment problem (5.1) has solutions $\phi \in \mathcal{S}(0, \infty)$ if and only if $\left|\alpha_{n}\right| \rightarrow \infty$ and there is $\sigma_{0}>0$ such that

(I) if $\lim _{n \rightarrow \infty} \alpha_{n}=\infty$, then (5.4) holds;

(II) if $\lim _{n \rightarrow \infty} \alpha_{n}=-\infty$, then (5.5) holds;

(III) if $\liminf _{n \rightarrow \infty} \alpha_{n}=-\infty$ and $\limsup _{n \rightarrow \infty} \alpha_{n}=\infty$, then both (5.4) and (5.5) hold.

Proof. We only need to show the converse. We assume $\lim _{n \rightarrow \infty} \Re e \alpha_{n}=\infty$, the proofs in remaining two cases are analogous. We may rearrange the sequence in increasing order $\alpha_{0}<\alpha_{1}<\cdots<\alpha_{n} \rightarrow \infty$. We prove that (5.4) implies (P2), for an extension sequence of the $x^{\alpha_{n}} \mathrm{~d} F(x)$ to $\mathcal{S}_{+}^{\prime}$, by contraposition. Note that the $O$-bound (5.6) holds as $x \rightarrow 0^{+}$with $-M=\alpha_{0}$; so, if (P2) fails, there are $m, \alpha>0$, and an infinite sequence of indices $N_{k}$ such that

$$
\int_{1}^{x}\left(t^{\alpha_{N_{k}}}+\sum_{n=0}^{N_{k}-1} b_{n, k} t^{\alpha_{n}}\right)\left(1-\frac{t}{x}\right)^{m} \mathrm{~d} F(t)=O\left(x^{\alpha}\right), \quad x \geq 1 .
$$

for some constants $b_{n, k}$. We have $\sum_{n=0}^{N_{k}-1} b_{n, k} t^{\alpha_{n}}=o\left(t^{\alpha_{N_{k}}}\right)$. Therefore,

$$
G(t)=\int_{1}^{x} t^{\alpha_{N_{k}}} \mathrm{~d} F(t) \leq 2^{m} \int_{1}^{2 x}\left(1-\frac{t}{2 x}\right)^{m} t^{\alpha_{N_{k}}} \mathrm{~d} F(t)=O\left(x^{\alpha}\right), \quad x \geq 1 .
$$

Integrating by parts,

$$
\int_{1}^{x} t^{\alpha_{N_{k}}-\alpha-1} \mathrm{~d} F(t)=\frac{G(x)}{x^{\alpha+1}}+(\alpha+1) \int_{1}^{x} \frac{G(t)}{t^{\alpha+2}} \mathrm{~d} t=O(1)
$$

which implies that (5.4) cannot hold because $\alpha_{N_{k}} \rightarrow \infty$.

If the measure $\mathrm{d} F$ vanishes on $\left(0, \lambda_{0}\right)$, then (5.5) cannot hold, which excludes the cases (II) and (III) from Proposition 5.1 and Theorem 5.2. Also, the abscissa of absolute convergence of the Mellin transform of $\int_{0}^{\infty} x^{-s} \mathrm{~d} F(x)$, denoted as $\sigma_{a}$, is the infimum of 
those $\sigma_{1}$ for which (5.3) holds. Under our assumption (5.3), $\sigma_{a}<\infty$, but it may be equal to $-\infty$.

Corollary 5.3. Let $F$ be a non-decreasing function of at most polynomial growth that vanish on $(-\infty, \lambda)$ for some $\lambda>0$, and let $\left\{\alpha_{n}\right\}_{n=0}^{\infty}$ be a sequence of distinct real numbers. Then, every moment problem (5.1) has solutions $\phi \in \mathcal{S}(0, \infty)$ if and only if $\alpha_{n} \rightarrow \infty$ and the abscissa of convergence of the Mellin transform of $\mathrm{d} F$ is finite, that is, $\sigma_{a}>-\infty$.

Example 5.4. Let $\left\{\lambda_{k}\right\}_{k=1}^{\infty}$ be a non-decreasing sequence of positive real numbers and let $\left\{c_{k}\right\}_{k=0}^{\infty}$ be a non-negative sequence. According to Corollary 5.3, every arbitrary moment problem

$$
a_{n}=\sum_{k=1}^{\infty} c_{k} \lambda_{k}^{\alpha_{n}} \phi(k), \quad n \in \mathbb{N} .
$$

has solutions $\phi \in \mathcal{S}(0, \infty)$ if and only if $\alpha_{n} \rightarrow \infty$ and the Dirichlet series $F(s)=$ $\sum_{k=1}^{\infty} c_{k} \lambda^{-s}$ has finite abscissa of convergence. In particular, if $\alpha_{n} \rightarrow \infty$, moment problems such as

$$
a_{n}=\sum_{k=1}^{\infty} \phi(k) k^{\alpha_{n}}, \quad n \in \mathbb{N}, \quad \text { and } \quad a_{n}=\sum_{p \text { prime }} \phi(p) p^{\alpha_{n}}, \quad n \in \mathbb{N},
$$

are always solvable in $\mathcal{S}(0, \infty)$.

\section{Density of the SET OF SOlutions of MOMENT PROBlems}

In order to study vector valued moment problems, it is convenient to consider first several results on the density of some linear manifolds in a general topological vector space.

Let $E$ be a locally convex topological vector space. It is well known that a linear functional $f: E \longrightarrow \mathbb{C}$ is continuous if and only if the linear subspace ker $f=\{x \in$ $E: f(x)=0\}$ is closed, or, equivalently, $f$ is discontinuous if and only if ker $f$ is dense in $E$. We would like to consider the corresponding situation when not one but several linear functionals are given. In the following we shall employ the notation $\operatorname{ker}\left(f_{1}, \ldots, f_{n}\right)=\bigcap_{k=1}^{n} \operatorname{ker} f_{k}$.

Definition 6.1. Let $f_{1}, \ldots, f_{n}$ be linear functionals on the locally convex topological vector space $E$. We say that they are completely discontinuous if the only linear combination $\sum_{k=1}^{n} c_{k} f_{k}$ that is continuous is the one with $c_{k}=0$ for all $k$.

If we denote by $\pi$ the projection from the algebraic dual space $E_{\text {alg }}^{\prime}$ onto $E_{\text {alg }}^{\prime} / E^{\prime}$, then $f_{1}, \ldots, f_{n}$ are completely discontinuous if and only if $\pi\left(f_{1}\right), \ldots, \pi\left(f_{n}\right)$ are linearly independent. 
Proposition 6.2. Let $f_{1}, \ldots, f_{n}$ be $n$ linearly independent linear functionals on the locally convex topological vector space $E$. Let $k$ be the dimension of the vector subspace $G$ of $\mathbb{C}^{n}$ formed by those vectors $\left(c_{1}, \ldots, c_{n}\right)$ such that $\sum_{i=1}^{n} c_{i} f_{i}$ is continuous. Then

$$
\operatorname{codim}_{E} \overline{\operatorname{ker}\left(f_{1}, \ldots, f_{n}\right)}=k \text {. }
$$

In particular, $\operatorname{ker}\left(f_{1}, \ldots, f_{n}\right)$ is dense in $E$ if and only if $f_{1}, \ldots, f_{n}$ are completely discontinuous.

Proof. Indeed, denote by $m$ the codimension of $\overline{\operatorname{ker}\left(f_{1}, \ldots, f_{n}\right)}$ in $E$. We shall first show that $m \geq k$. This is obvious, of course, if $k=0$, so let us suppose that $k>0$. Then if $\mathbf{c}_{j}=\left(c_{j, i}\right)_{i=1}^{n}, 1 \leq j \leq k$, are a basis of $G$, then the $k$ functionals $g_{j}=\sum_{i=1}^{n} c_{j, i} f_{i}$, $1 \leq j \leq k$ are continuous, and they are also linearly independent, because the $f_{j}$ 's are. Since $\operatorname{ker}\left(f_{1}, \ldots, f_{n}\right) \subset \operatorname{ker}\left(g_{1}, \ldots, g_{k}\right)$, and the latter space is closed, we obtain that $\overline{\operatorname{ker}\left(f_{1}, \ldots, f_{n}\right)} \subset \operatorname{ker}\left(g_{1}, \ldots, g_{k}\right)$, and thus $m \geq k$.

To show that $m \leq k$ we may assume that $m>0$. Since $\overline{\operatorname{ker}\left(f_{1}, \ldots, f_{n}\right)}$ is a closed subspace of codimension $m$, we can find $m$ linearly independent continuous functionals $g_{1}, \ldots, g_{m}$ such that $\overline{\operatorname{ker}\left(f_{1}, \ldots, f_{n}\right)}=\operatorname{ker}\left(g_{1}, \ldots, g_{m}\right)$. The fact that $\operatorname{ker}\left(f_{1}, \ldots, f_{n}\right) \subset$ ker $g_{j}$ for any $j$ yields that $g_{j}$ is a linear combination of $f_{1}, \ldots, f_{n}$, say $g_{j}=\sum_{i=1}^{n} c_{j, i} f_{i}$; then the vectors $\mathbf{c}_{j}=\left(c_{j, i}\right)_{i=1}^{n}$ for $1 \leq j \leq m$ are linearly independent in $G$, and thus $m \leq k$.

Observe, furthermore, that if $f_{1}, \ldots, f_{n}$ are linearly independent, then the dimension of the vector space generated by $\pi\left(f_{1}\right), \ldots, \pi\left(f_{n}\right)$ is precisely $n-k$.

If $f_{1}, \ldots, f_{n}$ are linearly independent, then the map from $E$ to $\mathbb{C}^{n}$ given by $x \mapsto$ $\left(\left\langle f_{j}, x\right\rangle\right)_{j=1}^{n}$ is surjective. Therefore we obtain the following result on finite moment problems.

Corollary 6.3. If $f_{1}, \ldots, f_{n}$ are completely discontinuous, then for any vector $\left(a_{j}\right)_{j=1}^{n}$ of $\mathbb{C}^{n}$, the set of solutions of the moment problem

$$
\left\langle f_{j}, x\right\rangle=a_{j}, \quad 1 \leq j \leq n,
$$

is dense in $E$.

Sometimes it is necessary to employ the following simple result.

Lemma 6.4. Let $E$ be a locally convex topological vector space and let $F$ be a closed subspace of finite codimension. Then the linear functionals $f_{1}, \ldots, f_{n}$ are completely discontinuous in $E$ if and only if their restrictions to $F$ are.

In general the intersection of an infinite sequence of dense linear manifolds does not have to be dense, so that the result of the Corollary 6.3 does not hold for infinite moment problems. We shall show that when $E=\mathcal{S}(0, \infty)$ and the linear functionals are Cesàro admissible then the density of the set of solutions holds in many cases, but before we do this we give an example when the set of solutions is not dense. 
Example 6.5. Let $E$ be the normed space formed by the polynomials in one variable, with norm $\|p\|=\max _{|t| \leq 1} p(t)$. Consider the sequence of functionals $\left\{f_{k}\right\}_{k=1}^{\infty}$, $f_{k}(t)=\delta^{(k)}(t)$. For each $n, f_{1}, \ldots, f_{n}$ are completely discontinuous, and as the corollary predicts, the set $S_{n}=\left\{p \in E:\left\langle f_{j}, p\right\rangle=a_{j}, 1 \leq j \leq n\right\}$ is dense in $E$ for any constants $a_{1}, \ldots, a_{n}$. However, for many infinite sequences $\left\{a_{k}\right\}_{k=1}^{\infty}$ we have that the set $\left\{p \in E:\left\langle f_{j}, p\right\rangle=a_{j}, j \geq 1\right\}$ is empty, and when not empty, it is an affine subspace of dimension 1. Thus $\bigcap_{n=1}^{\infty} S_{n}$ is never dense in $E$.

Suppose now that $E$ is a Fréchet space whose topology is given by the basis of increasing seminorms $\left\{p_{k}\right\}_{k=1}^{\infty}$. Consider a family $\left\{f_{k}\right\}_{k=1}^{\infty}$ of continuous linear functionals. We shall say that the sequence of blocks $\left\{\left\{f_{j}\right\}_{j=N_{k}}^{N_{k}+1}\right\}_{k=0}^{\infty}$ is strictly admissible with respect to the sequence of seminorms $\left\{p_{k}\right\}_{k=1}^{\infty}$ if $\left\{N_{k}\right\}_{k=0}^{\infty}$ is an increasing sequence of integers with $N_{0}=1$ such that the following two conditions are satisfied:

(1) $\left\{f_{1}, \ldots, f_{N_{k}}\right\}$ are continuous with respect to $p_{k}$;

(2) $\left\{f_{N_{k}+1}, f_{N_{k}+2}, \ldots\right\}$ are completely discontinuous with respect to $p_{k}$.

We can then prove the density of the set of solutions of certain infinite moment problems.

Proposition 6.6. Let $\left\{\left\{f_{j}\right\}_{j=N_{k}}^{N_{k}+1}\right\}_{k=0}^{\infty}$ be strictly admissible with respect to $\left\{p_{k}\right\}_{k=1}^{\infty}$. Let $\left\{a_{k}\right\}_{k=N_{1}+1}^{\infty}$ be an arbitrary sequence of complex numbers. Then the set of solutions of the moment problem

$$
\left\langle f_{j}, x\right\rangle=a_{j}, \quad j \geq N_{1}+1,
$$

is dense in the seminormed space $\left(E, p_{1}\right)$. Actually for any $\varepsilon>0$ and any $x_{0} \in E$ there is a solution of (6.1) such that

$$
p_{1}\left(x-x_{0}\right) \leq \varepsilon, \quad\left\langle f_{j}, x\right\rangle=\left\langle f_{j}, x_{0}\right\rangle, 1 \leq j \leq N_{1} .
$$

Proof. Let $\left\{\varepsilon_{k}\right\}_{k=1}^{\infty}$ be a sequence with $\varepsilon_{k}>0$ for all $k$ and with $\sum_{k=1}^{\infty} \varepsilon_{k}=\varepsilon$. Considering the set $f_{N_{1}+1}, \ldots, f_{N_{2}}$, which is completely discontinuous with respect to $p_{1}$, the Corollary 6.3 and the Lemma 6.4 show the existence of $x_{1}$ such that $p_{1}\left(x_{1}\right)<\varepsilon_{1}$, $\left\langle f_{j}, x_{1}\right\rangle=0$ for $1 \leq j \leq N_{1}$, while $\left\langle f_{j}, x_{1}\right\rangle=a_{j}-\left\langle f_{j}, x_{0}\right\rangle$ for $N_{1}+1 \leq j \leq N_{2}$. Proceeding in a recursive fashion, for each $k \geq 2$ we can find $x_{k}$ such that $p_{k}\left(x_{k}\right)<\varepsilon_{k}$, $\left\langle f_{j}, x_{k}\right\rangle=0$ for $1 \leq j \leq N_{k}$, while $\left\langle f_{j}, x_{k}\right\rangle=a_{j}-\sum_{i=0}^{k-1}\left\langle f_{j}, x_{i}\right\rangle$ for $N_{k}+1 \leq j \leq N_{k+1}$.

The series $\sum_{k=0}^{\infty} x_{k}$ converges in $E$ since for any $q$ we have

$$
\sum_{k=0}^{\infty} p_{q}\left(x_{k}\right) \leq \sum_{k=0}^{q-1} p_{q}\left(x_{k}\right)+\sum_{k=q}^{\infty} p_{q}\left(x_{k}\right) \leq \sum_{k=0}^{q-1} p_{q}\left(x_{k}\right)+\sum_{k=q}^{\infty} \varepsilon_{k}<\infty .
$$


Let $x=\sum_{k=0}^{\infty} x_{k}$. Then

$$
p_{1}\left(x-x_{0}\right) \leq \sum_{k=1}^{\infty} p_{1}\left(x_{k}\right) \leq \sum_{k=1}^{\infty} \varepsilon_{k}=\varepsilon,
$$

while by continuity $\left\langle f_{j}, x\right\rangle=\sum_{i=0}^{\infty}\left\langle f_{j}, x_{i}\right\rangle$, which is actually a finite sum; this gives gives $\left\langle f_{j}, x\right\rangle=\left\langle f_{j}, x_{0}\right\rangle$, for $1 \leq j \leq N_{1}$ and if $k \geq 1$,

$$
\left\langle f_{j}, x\right\rangle=\sum_{i=0}^{k}\left\langle f_{j}, x_{i}\right\rangle=\left\langle f_{j}, x_{k}\right\rangle+\sum_{i=0}^{k-1}\left\langle f_{j}, x_{i}\right\rangle=a_{j},
$$

for $N_{k}+1 \leq j \leq N_{k+1}$, as required.

Let us now go back to moment problems in the space $E=\mathcal{S}(0, \infty)$. If a sequence of functionals $\left\{f_{k}\right\}_{k=0}^{\infty}$ is the restriction to $(0, \infty)$ of a sequence that is Cesàro admissible, and $\left\{p_{k}\right\}_{k=1}^{\infty}$ is an increasing sequence of continuous seminorms of $E$ that gives the topology, in general it is not possible to arrange $\left\{f_{k}\right\}_{k=0}^{\infty}$ in blocks to obtain strict admissibility. However, we can construct sequences $\left\{\widetilde{p}_{k}\right\}_{k=1}^{\infty},\left\{g_{k}\right\}_{k=0}^{\infty}$, and $\left\{N_{k}\right\}_{k=0}^{\infty}$ such that

(1) $\left\{\widetilde{p}_{k}\right\}_{k=1}^{\infty}$ is also an increasing sequence of continuous seminorms that gives the topology of $E$;

(2) $\left\{\left\{g_{j}\right\}_{j=N_{k}}^{N_{k}+1}\right\}_{k=0}^{\infty}$ is strictly admissible with respect to $\left\{\widetilde{p}_{k}\right\}_{k=1}^{\infty}$;

(3) there is a linear bijective map $T$ from $\mathbb{C}^{\mathbb{N}}$ to itself ${ }^{5}$ such that $T\left(\left\{f_{j}\right\}_{j=0}^{\infty}\right)$ $=\left\{g_{j}\right\}_{j=0}^{\infty}$. Actually there are increasing sequences $m_{0}=0<m_{1}<m_{2}<\cdots$, such that the vector $\left(g_{j}\right)_{j=m_{k}+1}^{m_{k+1}}$ is obtained by multiplying $\left(f_{j}\right)_{j=m_{k}+1}^{m_{k+1}}$ by an invertible matrix.

The construction is as follows. Naturally we put $N_{0}=1$. Next, take $\widetilde{p}_{1}=p_{1}$. Since $\left\{f_{k}\right\}_{k=0}^{\infty}$ is Cesàro admissible, we can find integers $n_{1}$ and $l_{1}$, with $n_{1} \leq l_{1}$ such that $f_{0}, \ldots, f_{n_{1}}$ are continuous with respect to $\widetilde{p}_{1}$ while $f_{l_{1}+1}, f_{l_{1}+2}, f_{l_{1}+3}, \ldots$ are completely discontinuous with respect to $\widetilde{p}_{1}$; we can take them so that $n_{1}$ is the maximum while $l_{1}$ is the minimum with this property. If $l_{1}=n_{1}$ we take $N_{1}=n_{1}$. If $l_{1}=n_{1}+q, q \geq 1$, we can find linear combinations

$$
g_{n_{1}+j}=\sum_{i=1}^{q} \alpha_{j, i} f_{n_{1}+i}+\sum_{k=n_{1}+q+1}^{m_{1}} \beta_{j, i} f_{k}
$$

for $1 \leq j \leq q$ such that the matrix $\left(\alpha_{j, i}\right)_{j, i=1}^{q}$ is invertible, and for some $N_{1}$, with $n_{1}<N_{1} \leq l_{1}$ the functionals $f_{0}, \ldots, f_{n_{1}}, g_{n_{1}+1}, \ldots g_{N_{1}}$ are continuous with respect to $\widetilde{p}_{1}$ while $g_{N_{1}+1}, \ldots, g_{l_{1}}, f_{l_{1}+1}, f_{l_{1}+2}, f_{l_{1}+3}, \ldots$ are completely discontinuous with respect

\footnotetext{
${ }^{5}$ The map $T$ is actually bicontinuous with respect to the topology of pointwise convergence in $\mathbb{C}^{\mathbb{N}}$.
} 
to $\widetilde{p}_{1}$. Put $g_{j}=f_{j}$ for $0 \leq j \leq n_{1}$ and for $l_{1}+1 \leq j \leq m_{1}$. The vector $\left(g_{j}\right)_{j=1}^{m_{1}}$ is obtained by multiplying $\left(f_{j}\right)_{j=1}^{m_{1}}$ by an invertible matrix.

We then find a seminorm $\widetilde{p}_{2}$ among the $p_{k}$ 's for $k \geq r_{1}$ such that $g_{0}, \ldots, g_{m_{1}}$ and $f_{m_{1}+1}, \ldots, f_{n_{2}}$ are continuous with respect to $\widetilde{p}_{2}$ while $f_{l_{2}+1}, f_{l_{2}+2}, f_{l_{2}+3}, \ldots$ are completely discontinuous with respect to $\widetilde{p}_{2}$ for some integers $n_{2}$ and $l_{2}$, with $m_{1}+1 \leq n_{2} \leq$ $l_{2}$. Then we employ the same procedure to find $N_{2}$ and $m_{2}$ such that $n_{2} \leq N_{2} \leq l_{2} \leq m_{2}$ and linear combinations $g_{j}, m_{1}+1 \leq j \leq m_{2}$ of the $f_{k}$ 's, $m_{1}+1 \leq k \leq m_{2}$, so that $\left(g_{j}\right)_{j=m_{1}+1}^{m_{2}}$ is obtained by multiplying $\left(f_{j}\right)_{j=m_{1}+1}^{m_{2}}$ by an invertible matrix, $g_{0}, \ldots g_{N_{2}}$ are continuous with respect to $\widetilde{p}_{2}$ while $g_{N_{2}+1}, g_{N_{2}+2}, g_{N_{2}+3}, \ldots$ are completely discontinuous with respect to $\widetilde{p}_{2}$.

We may then proceed inductively, constructing seminorms $\widetilde{p}_{k}$, integers $n_{k}, N_{k}, l_{k}$, and $m_{k}$ with $m_{k-1}+1 \leq n_{k} \leq N_{k} \leq l_{k} \leq m_{k}$, define $\left(g_{j}\right)_{j=m_{k-1}+1}^{m_{k}}$ by multiplying $\left(f_{j}\right)_{j=m_{k-1}+1}^{m_{k}}$ by an appropriate invertible matrix, so that $g_{1}, \ldots g_{N_{k}}$ are continuous with respect to $\widetilde{p}_{k}$ while $g_{N_{k}+1}, g_{N_{k}+2}, g_{N_{k}+3}, \ldots$ are completely discontinuous with respect to $\widetilde{p}_{k}$. The three conditions above are then clearly satisfied.

Notice that condition $(\mathbf{3})$ in our construction implies that the moment problems

$$
\left\langle f_{j}, \phi\right\rangle=a_{j}, \quad j \in \mathbb{N},
$$

have solution for all sequences $\left\{a_{k}\right\}_{k=0}^{\infty}$ if and only if the same is true of all the moment problems

$$
\left\langle g_{j}, \phi\right\rangle=b_{j}, \quad j \in \mathbb{N},
$$

for arbitrary sequences $\left\{b_{k}\right\}_{k=0}^{\infty}$. We can also use the density of the set of solutions of one moment problem to obtain the corresponding density of the set of solutions of the other, as we explain in precise terms in the next proposition.

Proposition 6.7. Let $\left\{f_{k}\right\}_{k=0}^{\infty}$ be a sequence of distributions of the space $\mathcal{S}_{+}$that satisfies conditions $(\mathbf{P} 1)$ and $(\mathbf{P 2})$. Let $p$ be any continuous seminorm in $\mathcal{S}(0, \infty)$. Then there exists $m \in \mathbb{N}$ such that if $\left\{a_{k}\right\}_{k=m}^{\infty}$ is an arbitrary sequence of complex numbers, then the set of solutions of the moment problem

$$
\left\langle f_{j}, \phi\right\rangle=a_{j}, \quad j \geq m
$$

is dense in the seminormed space $(\mathcal{S}(0, \infty), p)$. If the sequence $\left\{f_{k}\right\}_{k=0}^{\infty}$ is completely discontinuous with respect to $p$ then we can take $m=0$.

Proof. Let $\left\{p_{k}\right\}_{k=1}^{\infty}$ be an increasing sequence of continuous seminorms of $\mathcal{S}(0, \infty)$ that gives the topology, with $p_{1}=p$. We can then construct the sequence $\left\{g_{j}\right\}$ so that conditions $(\mathbf{1}),(\mathbf{2})$, and $(\mathbf{3})$ are satisfied. Observe that for any $k$ the set of solutions of the moment problem $\left\langle f_{j}, \phi\right\rangle=0$, for $j \geq m_{k}$, is exactly the same as the set of solutions of $\left\langle g_{j}, \phi\right\rangle=0$, for $j \geq m_{k}$, and since $\left\{\left\{g_{j}\right\}_{j=N_{k}}^{N_{k}+1}\right\}_{k=0}^{\infty}$ is strictly admissible with respect to $\left\{\widetilde{p}_{k}\right\}_{k=1}^{\infty}$, Proposition 6.6 gives that such set of solutions is dense in $\mathcal{S}(0, \infty)$ with 
the topology given by the seminorm $\widetilde{p}_{k}$. Hence, if we take $m=m_{1}$ we obtain that the set of solutions of $\left\langle f_{j}, \phi\right\rangle=0$ for $j \geq m$ is dense in $(\mathcal{S}(0, \infty), p)$. Therefore, if $\left\{a_{k}\right\}_{k=m}^{\infty}$ is an arbitrary sequence of complex numbers, by translating by a particular solution of (6.2) - particular solution that exists because $(\mathbf{P 1})$ and $(\mathbf{P} 2)$ are satisfied - we obtain that the set of solutions of $(6.2)$ is likewise dense in $(\mathcal{S}(0, \infty), p)$. That we can take $m=0$ if $\left\{f_{k}\right\}_{k=0}^{\infty}$ is completely discontinuous with respect to $p$ should be clear from our construction.

The next related result, which will be needed in our analysis of the vector moment problems, follows by the same arguments.

Proposition 6.8. Let $\left\{f_{k}\right\}_{k=0}^{\infty}$ be a sequence of distributions of the space $\mathcal{S}_{+}$that satisfies conditions $(\mathbf{P 1})$ and $(\mathbf{P 2})$. Let $p$ be any continuous seminorm in $\mathcal{S}(0, \infty)$. Then there exists $m \in \mathbb{N}$ such that if $\left\{a_{k}\right\}_{k=m}^{\infty}$ is an arbitrary sequence of complex numbers, then for each $\varepsilon>0$ the moment problem

$$
\left\langle f_{j}, \phi\right\rangle=a_{j}, \quad j \geq m, \quad\left\langle f_{j}, \phi\right\rangle=0, \quad 0 \leq j<m,
$$

has a solution with $p(\phi)<\varepsilon$.

\section{VECTOR MOMENT PROBLEMS}

We now consider some vector moment problems.

Let $\mathcal{X}$ be a Fréchet space. Let \|\|$_{1} \leq\|\|_{2} \leq\|\|_{3} \ldots$ be a sequence of seminorms of $\mathcal{X}$ that generate its topology. Let $\left\{\mathbf{a}_{k}\right\}_{k=0}^{\infty}$ be an arbitrary sequence of elements of $\mathcal{X}$ and let $\left\{f_{j}\right\}_{j=0}^{\infty}$ be a sequence of distributions of the space $\mathcal{S}_{+}$. We wish to study the problem of finding a rapidly decreasing smooth function $\phi: \mathbb{R} \rightarrow \mathcal{X}$ with support in $[0, \infty)$ such that

$$
\left\langle f_{j}, \phi\right\rangle=\mathbf{a}_{j}, \quad j=0,1,2,3, \ldots .
$$

Notice that asking $\phi$ to be a rapidly decreasing smooth function means that $\phi \in$ $\mathcal{S}(\mathbb{R}, \mathcal{X}) \cong \mathcal{S}(\mathbb{R}) \widehat{\otimes} \mathcal{X}$. In general [26], $\psi$ belongs to $\mathcal{S}\left(\mathbb{R}^{n}, \mathcal{X}\right)$ if and only if for each $\mathbf{k}, \mathbf{m} \in \mathbb{N}^{n}$ the set $\left\{\mathbf{x}^{\mathbf{k}} \mathbf{D}^{\mathbf{m}} \psi(\mathbf{x}): \mathbf{x} \in \mathbb{R}^{n}\right\}$ is bounded in $\mathcal{X}$. For a Fréchet space this means that

$$
\left\|\mathbf{D}^{\mathbf{m}} \psi(\mathbf{x})\right\|_{q}=O\left(|\mathbf{x}|^{-k}\right), \text { as }|\mathbf{x}| \rightarrow \infty,
$$

for each $\mathbf{m} \in \mathbb{N}^{n}$ and $q, k \in \mathbb{N}$. Notice also that $\mathcal{S}((0, \infty), \mathcal{X}) \cong \mathcal{S}(0, \infty) \widehat{\otimes} \mathcal{X}$ are the elements of $\mathcal{S}(\mathbb{R}, \mathcal{X})$ with support in $[0, \infty)$.

If $\left\{f_{j}\right\}_{j=0}^{\infty}$ is a sequence of distributions of the space $\mathcal{S}_{+}$that satisfies conditions $(\mathbf{P} \mathbf{1})$ and $(\mathbf{P} 2)$, then we can find functions $\rho_{k} \in \mathcal{S}(0, \infty)$ such that

$$
\left\langle f_{j}, \rho_{k}\right\rangle=\delta_{j, k}, \quad j=0,1,2,3, \ldots,
$$

where $\delta_{j, k}=0, j \neq k, \delta_{k, k}=1$, is the Kronecker delta. One is tempted to try to solve (7.1) by setting $\phi(x)=\sum_{k=0}^{\infty} \rho_{k}(x) \mathbf{a}_{k}$. However, this series could be divergent 
and even if convergent the sum might not belong to $\mathcal{S}((0, \infty), \mathcal{X})$. However, as we shall show, if we choose the $\rho_{k}$ 's carefully then the series converges and gives an element of $\mathcal{S}((0, \infty), \mathcal{X})$.

Theorem 7.1. Let $\mathcal{X}$ be a Fréchet space and let $\left\{\mathbf{a}_{k}\right\}$ be an arbitrary sequence of elements of $\mathcal{X}$. Let $\left\{f_{k}\right\}_{k=0}^{\infty}$ be a sequence of distributions of the space $\mathcal{S}_{+}$that satisfies conditions (P1) and (P2). Then the moment problem

$$
\left\langle f_{j}, \phi\right\rangle=\mathbf{a}_{j}, \quad j \in \mathbb{N} .
$$

has solutions $\phi \in \mathcal{S}((0, \infty), \mathcal{X})$.

Proof. Let

$$
Q_{n}=\left\|\mathbf{a}_{n}\right\|_{n},
$$

so that $\left\|\mathbf{a}_{j}\right\|_{n} \leq Q_{j}$ if $j \geq n$. Let us choose $\varepsilon_{n}>0$ so that $\sum_{n=1}^{\infty} \varepsilon_{n} Q_{n}<\infty$. Let $\left\{p_{k}\right\}_{k=1}^{\infty}$ be an increasing sequence of continuous seminorms of $\mathcal{S}(0, \infty)$ that gives the topology.

Employing Proposition 6.8 we can find a sequence of positive integers $\left\{m_{i}\right\}_{i=1}^{\infty}$, which we may suppose increasing, and for $k \geq m_{i}$ solutions $\rho_{k}^{\{i\}}$ of the moment problem (7.3) with $p_{i}\left(\rho_{k}^{\{i\}}\right) \leq \varepsilon_{k}$. If we now put $\rho_{k}=\rho_{k}^{\{i\}}$ for $m_{i} \leq k \leq m_{i+1}-1$, and take any solutions $\rho_{k}$ for $k<m_{1}$, then the series $\sum_{k=0}^{\infty} \rho_{k}(x) \mathbf{a}_{k}$ converges in $\mathcal{S}(0, \infty) \widehat{\otimes} \mathcal{X} \cong$ $\mathcal{S}((0, \infty), \mathcal{X})$. Indeed, convergence of the series in the tensor product would follow [26] if we show that for each $N$ and $M$ the series $\sum_{k=0}^{\infty} p_{N}\left(\rho_{k}\right)\left\|\mathbf{a}_{k}\right\|_{M}$ converges. But by taking $K=\max \left\{M, m_{N}\right\}$ we obtain

$$
\begin{aligned}
\sum_{k=0}^{\infty} p_{N}\left(\rho_{k}\right)\left\|\mathbf{a}_{k}\right\|_{M} & \leq \sum_{k=0}^{K-1} p_{N}\left(\rho_{k}\right)\left\|\mathbf{a}_{k}\right\|_{M}+\sum_{k=K}^{\infty} p_{N}\left(\rho_{k}\right)\left\|\mathbf{a}_{k}\right\|_{M} \\
& \leq \sum_{k=0}^{K-1} p_{N}\left(\rho_{k}\right)\left\|\mathbf{a}_{k}\right\|_{M}+\sum_{k=K}^{\infty} \varepsilon_{k} Q_{k}<\infty .
\end{aligned}
$$

If we now put $\phi(x)=\sum_{k=0}^{\infty} \rho_{k}(x) \mathbf{a}_{k}$ we obtain

$$
\left\langle f_{j}, \phi\right\rangle=\sum_{k=0}^{\infty}\left\langle f_{j}, \rho_{k}(x)\right\rangle \mathbf{a}_{k}=\sum_{k=0}^{\infty} \delta_{j, k} \mathbf{a}_{k}=\mathbf{a}_{j},
$$

because of the convergence and the continuity of the $f_{j}$ 's.

Needless to say, the conditions (P1) and (P2) are also necessary for the solvability of the vector moment problem, as follows from Theorem 3.1. 


\section{Moment PROBlems in SEVERAL VARIABLES}

In this section we consider moment problems for functions of several variables. Indeed, let $V$ be an open cone with vertex at the origin in $\mathbb{R}^{d}$, that is, $V$ is an open set such that $\lambda \mathbf{x} \in V$ whenever $\lambda>0$ and $\mathbf{x} \in V$. Let $\mathcal{X}$ be a Fréchet space. We denote by $\mathcal{S}(V, \mathcal{X})$ the space of elements of $\mathcal{S}\left(\mathbb{R}^{d}, \mathcal{X}\right)$ with support contained in $\bar{V}$. If $\left\{F_{n}\right\}_{n=0}^{\infty}$ is a sequence of distributions of the space $\mathcal{S}^{\prime}\left(\mathbb{R}^{d}\right)$ and $\left\{\mathbf{a}_{n}\right\}_{n=0}^{\infty}$ is a sequence of vectors of $\mathcal{X}$, we would like to study the existence of solutions of the vector moment problem

$$
\left\langle F_{n}(\mathbf{x}), \phi(\mathbf{x})\right\rangle=\mathbf{a}_{n}, \quad n \in \mathbb{N},
$$

in the space $\mathcal{S}(V, \mathcal{X})$.

We shall denote by $\mathbb{S}$ the unit sphere of $\mathbb{R}^{d}$, and if $U$ is an open subset of $\mathbb{S}$ then $\mathcal{D}(U)$ will denote the set of smooth functions defined in $\mathbb{S}$ whose support is contained in $U$.

If $F \in \mathcal{S}^{\prime}\left(\mathbb{R}^{d}\right)$ and $\psi$ is a smooth function defined in $\mathbb{S}$ then we can define the distribution $f(r)=\langle F(r \omega), \psi(\omega)\rangle_{\omega}$ of the space $\mathcal{S}^{\prime}(0, \infty)$ by

$$
\langle f(r), \rho(r)\rangle_{r}=\left\langle F_{n}(\mathbf{x}),|\mathbf{x}|^{1-d} \rho(|\mathbf{x}|) \psi(\mathbf{x} /|\mathbf{x}|)\right\rangle, \quad \rho \in \mathcal{S}(0, \infty) .
$$

In general this equation cannot be applied if $\rho \in \mathcal{S}_{+}$, so that $f$ does not belong to $\mathcal{S}_{+}^{\prime}$, but there are always extensions ${ }^{6}$ of $f$ to $\mathcal{S}_{+}^{\prime}$.

Proposition 8.1. Let $\left\{F_{n}\right\}_{n=0}^{\infty}$ be a sequence of distributions of the space $\mathcal{S}^{\prime}\left(\mathbb{R}^{d}\right)$. Suppose there exists a smooth function $\psi \in \mathcal{D}(V \cap \mathbb{S})$ such that the generalized functions of one variable $f_{n}(r)=\left\langle F_{n}(r \omega), \psi(\omega)\right\rangle_{\omega}$ have extensions ${ }^{7}$ in $\mathcal{S}_{+}^{\prime}$ that satisfy $(\mathbf{P} \mathbf{1})$ and (P2). Then for any arbitrary sequence of vectors $\left\{\mathbf{a}_{n}\right\}_{n=0}^{\infty}$ in a Fréchet space $\mathcal{X}$ the moment problem (8.1) has solutions in $\mathcal{S}(V, \mathcal{X})$.

Proof. Indeed, the moment problem $\left\langle f_{n}(r), \varphi(r)\right\rangle=\mathbf{a}_{n}$ has solutions $\varphi \in \mathcal{S}((0, \infty), \mathcal{X})$. If we put

$$
\phi(\mathbf{x})=|\mathbf{x}|^{1-d} \varphi(|\mathbf{x}|) \psi(\mathbf{x} /|\mathbf{x}|)
$$

then $\phi \in \mathcal{S}(V, \mathcal{X})$ and it satisfies the moment problem (8.1).

Proposition 8.1 implies that if the $F_{n}$ 's are radial distributions, that is, they depend only on $|\mathbf{x}|, F_{n}(\mathbf{x})=f_{n}(|\mathbf{x}|)$ and the distributions ${ }^{8} f_{n}$ satisfy $(\mathbf{P 1})$ and $(\mathbf{P} 2)$, then (8.1) has solutions in $\mathcal{S}(V, \mathcal{X})$ for any cone $V$ : we may just take $\psi$ as any element of $\mathcal{S}(V \cap \mathbb{S})$ whose integral over $V \cap \mathbb{S}$ does not vanish. Actually we can improve this result:

\footnotetext{
${ }^{6}$ Interestingly [9], there are no continuous extension operators from $\mathcal{S}^{\prime}(0, \infty)$ to $\mathcal{S}_{+}^{\prime}$.

${ }^{7}$ These distributions belong to the spaces $\mathcal{R}_{d}$ introduced in [15].

${ }^{8}$ The distributions of one variable $f_{n}$ are not unique [10].
} 
Corollary 8.2. Let $\mathcal{X}$ be a Fréchet space and let $\left\{\mathbf{a}_{k}\right\}$ be an arbitrary sequence of elements of $\mathcal{X}$. Let $\left\{f_{n}\right\}_{n=0}^{\infty}$ be a sequence of distributions of $\mathcal{S}_{+}^{\prime}$ for which (P1) and (P2) hold and let $\left\{g_{n}\right\}_{n=0}^{\infty}$ be a distribution sequence in $\mathcal{D}^{\prime}(\mathbb{S})$. If $\left\{F_{n}\right\}_{n=0}^{\infty}$ is any sequence of distributions in $\mathcal{S}^{\prime}\left(\mathbb{R}^{d}\right)$ such that

$$
\left\langle F_{n}(\mathbf{x}), \phi(\mathbf{x})\right\rangle=\left\langle f_{n}(r) \otimes g_{n}(\omega), r^{d-1} \phi(r \omega)\right\rangle \quad \text { for each } \phi \in \mathcal{S}(V),
$$

then (8.1) is solvable in $\mathcal{S}(V, \mathcal{X})$ for any open cone $V$ provided that there is an open set $U$ of $\mathbb{S}$ such that $\bar{U} \subset V \cap \mathbb{S}$ and $g_{n} \neq 0$ on $U$.

Proof. In fact, using Proposition 8.1, it suffices to check that there is a smooth function $\psi$ with $\operatorname{supp} \psi \subseteq \bar{U}$ such that $\left\langle g_{n}(\omega), \psi(\omega)\right\rangle \neq 0$ for all $n \in \mathbb{N}$. To show this, consider

the Fréchet space $\mathcal{Y}=\left\{\psi \in C^{\infty}(\mathbb{S}): \operatorname{supp} \psi \subseteq \bar{U}\right\}$ and the sequence of closed subspaces $\mathcal{Y}_{n}=\operatorname{ker}_{\mathcal{Y}} g_{n}=\left\{\psi \in \mathcal{Y}:\left\langle g_{n}, \psi\right\rangle=0\right\}$. Since each $g_{n}$ is non-identically zero on $U$, we have that $\mathcal{Y}_{n} \neq \mathcal{Y}$ is of the first category. The Baire theorem implies that $\mathcal{Y} \neq \bigcup_{n=0}^{\infty} \mathcal{Y}_{n}$

\section{REFERENCES}

[1] R. P. Boas, The Stieltjes moment problem for functions of bounded variation, Bull. Amer. Math. Soc. 45 (1939), 399-404.

[2] J. Campos Ferreira, Introduction to the theory of distributions, Longman, Harlow, 1997.

[3] J. Chung, S. -Y. Chung, D. Kim, Every Stieltjes moment problem has a solution in Gel'fand-Shilov spaces, J. Math. Soc. Japan 55 (2003), 909-913.

[4] A. J. Durán, The Stieltjes moments problem for rapidly decreasing functions, Proc. Amer. Math. Soc. 107 (1989), 731-741.

[5] A. J. Durán, The Stieltjes moment problem with complex exponents, Math. Nachr. 158 (1992), $175-194$.

[6] A. L. Durán, R. Estrada, Strong moment problems for rapidly decreasing smooth functions, Proc. Amer. Math. Soc. 120 (1994), 529-534.

[7] R. Estrada, Vector moment problems for rapidly decreasing smooth functions of several variables, Proc. Amer. Math. Soc. 126 (1998), 761-768.

[8] R. Estrada, The Cesàro behaviour of distributions, R. Soc. Lond. Proc. Ser. A Math. Phys. Eng. Sci. 454 (1998), 2425-2443.

[9] R. Estrada, The non-existence of regularization operators, J. Math. Anal. Appl. 286 (2003), 1-10.

[10] R. Estrada, On radial functions and distributions and their Fourier transforms, J. Fourier Anal. Appl. 20 (2014), 301-320.

[11] R. Estrada, R. P. Kanwal, A distributional approach to asymptotics. Theory and applications, Second edition, Birkhäuser Boston, Inc., Boston, MA, 2002.

[12] R. Estrada, J. Vindas, A general integral, Dissertationes Math. 483 (2012), 1-49.

[13] R. Estrada, J. Vindas, On Tauber's second Tauberian theorem, Tohoku Math. J. 64 (2012), 539-560.

[14] F. Galindo, F. López, J. Sanz, A generalized moment problem for rapidly decreasing smooth vector functions of several variables, J. Math. Anal. Appl. 263 (2001), 655-665.

[15] L. Grafakos, G. Teschl, On Fourier transforms of radial functions and distributions, J. Fourier Anal. Appl. 19 (2013), 167-179. 
[16] G. Köthe, Topological vector spaces. II, Springer-Verlag, New York-Berlin, 1979.

[17] A. Lastra, J. Sanz, Linear continuous operators for the Stieltjes moment problem in GelfandShilov spaces, J. Math. Anal. Appl. 340 (2008), 968-981.

[18] A. Lastra, J. Sanz, Stieltjes moment problem in general Gelfand-Shilov spaces, Studia Math. 192 (2009), 111-128.

[19] S. Łojasiewicz, Sur la valuer et la limite d'une distribution en un point, Studia Math. 16 (1957), $1-36$.

[20] S. Pilipović, B. Stanković, J. Vindas, Asymptotic behavior of generalized functions, Series on Analysis, Applications and Computations, 5, World Scientific Publishing Co., Hackensack, NJ, 2012.

[21] G. Pólya, Sur l'indetermination d'un théorème voisin du probleme des moments, C.R. Acad. Sci. Paris 207 (1938), 708-711.

[22] A. P. Robertson, W. Robertson, Topological vector spaces, Cambridge University Press, LondonNew York, 1973.

[23] J. Sebastião e Silva, Su certe classi di spazi localmente convessi importanti per le applicazioni, Rend. Mat. e Appl. (5) 14 (1955), 388-410.

[24] J. A. Shohat, J. D. Tamarkin, The problem of moments, American Mathematical Society Mathematical Surveys, vol. I, AMS, New York, 1943.

[25] T. J. Stieltjes, Recherches sur les fractions continues, Reprint of the 1894 original, Ann. Fac. Sci. Toulouse Math. (6) 4 (1995), pp. 1-35 \& 36-75.

[26] F. Trèves, Topological vector spaces, distributions and kernel, Academic Press, New York, 1967.

[27] J. Vindas, R. Estrada, On the support of tempered distributions, Proc. Edin Math. Soc. 53 (2010), $255-270$.

[28] V. S. Vladimirov, Methods of the theory of generalized functions, Analytical Methods and Special Functions, 6, Taylor \& Francis, London, 2002.

R. Estrada, Department of Mathematics, Louisiana State University, Baton Rouge, LA 70803, U.S.A.

E-mail address: restrada@math.1su.edu

J. Vindas, Department of Mathematics, Ghent University, Krijgslaan 281, B 9000 Gent, Belgium

E-mail address: jasson.vindas@UGent.be 